\title{
La territorialidad virtual en la creación participativa: el mapa interactivo y sus paisajes como forma de comunicación
}

\author{
Virtual territoriality in collaborative creation: \\ interactive map and its landscapes as a form of \\ communication
}

Juanjo Balaguer - Universidad de Granada- balaguerjuanjo@gmail.com

\section{Resumen:}

De entre la multitud de herramientas, procesos y tecnologías que estructuran nuevas narrativas en el entorno virtual, los mapas interactivos han ocupado un espacio significativo. Esta técnica consiste en una interfaz que permite tanto la navegabilidad en el mapa como, en ocasiones, la contribución por parte de cualquier usuario, a veces con geolocalización.

Aunque estos mapas se han creado a menudo con una función periodística, también han conformado otro tipo de iniciativas. Con indicación previa sobre la temática o la finalidad del mapa, los usuarios están habilitados en esos últimos casos para subir contenidos que, dependiendo del proyecto, pueden ser sonoros, gráficos, textuales o audiovisuales.

El presente trabajo analizará una serie de creaciones cuya narración polifónica y dispersa se organiza en torno a la representación gráfica de un territorio. Se considerará un caso temprano (One Day on Earth, 2012) y otros más recientes (Queering the Map, 2017; Radio Right Left, 2017).

Los archivos que se diseminan por esta interfaz cartográfica constituyen una suerte de paisajes de los lugares que habitan los usuarios. En su representación virtual, estos espacios son apropiados para convertirse en un territorio diverso y abierto, en consonancia con la lógica de la cultura participativa y de la sociedad red. Por ello, esta modalidad de participación 


\section{Las nuevas narrativas, en el entorno social Universidad de La Laguna, diciembre de 2019}

tiene un condicionante a priori que permitiría expresar una pluralidad no siempre presente en otras formas de creación colectiva.

En este sentido, la territorialidad simbólica del mapa interactivo podría tener implicaciones en relación con la visibilidad de experiencias locales, representaciones sociales, identidades colectivas, así como con expresiones de pertenencia o desarraigo.

\section{Abstract:}

Among the variety of tools, processes and technologies that structure new narratives on the internet, interactive maps have had a relevant position. This interface allows navigability as well as, sometimes, collaboration through users' contributions.

Although these maps have often been created with a journalistic purpose, they have shaped other types of projects. In some of these, users can contribute with their own contents with prior indication of subject, purpose, and media, which, depending on the project, can be text, sound, graphic or audiovisual archives.

This work analyses selected works whose collective structure is organised around the graphic representation of a territory. An early case (One Day on Earth, 2012) and two more recent projects (Queering the Map, 2017; Radio Right Left, 2017) will be considered.

The contributions dispersed around the map form a sort of landscape of these places that users inhabit. In these virtual representations, these spaces are appropriated to become a plural territory in line with the logic of participatory culture and network society. Hence, this modality of participation has a conditioning factor that could mean more plurality than other forms of collaborative creation.

Consequently, the symbolic territoriality of collaborative maps could have implications concerning more exposure for local experiences, social representations, collective identities, as well as expressions of uprooting or belonging. 


\section{Las nuevas narrativas, en el entorno social \\ Universidad de La Laguna, diciembre de 2019}

Palabras clave: mapa colaborativo; creación participativa; comunicación digital; espacio virtual; comunidad.

Keywords: collaborative map; participatory creation; digital communication; virtual space; community.

\section{Introducción: mapas digitales, interactividad y colaboración}

Al mismo tiempo que el desarrollo de la comunicación asume su virtualización, una parte de la vasta producción digital busca evidenciar los lugares donde los sujetos se expresan a través de técnicas creativas que recuperan la fisicidad característica del mapa. Interfaces interactivas formadas por una representación cartográfica estructuran multitud de iniciativas de distinto orden, mientras la geolocalización permite que la información que ocupa esos mapas esté anclada al espacio geográfico en que se emite.

La experiencia de los usuarios sigue marcada por la interactividad, de manera que navegan por los mapas y clican allá donde se ofrece información. En ocasiones pueden además ubicar su propio contenido. Ahora digital e interactivo, el mapa cambia su estética y su funcionalidad: se basa en la fragmentación, hipertextualidad y apertura características de la red. Recupera simbólicamente el territorio, con un "anclaje a unas determinadas coordenadas espaciales, simbólicas y temporales de la producción estética material, física y objetual" frente al carácter "desterritorializado" de la creación en los entornos digitales (Alberich-Pascual y Gómez-Pérez, 2017: 16).

El mapa interactivo ha dado forma a iniciativas en multitud de ámbitos. Investigaciones previas (Renó y Renó, 2013, 2015) destacaron su relevancia para el periodismo. El carácter lúdico del formato facilitaría, según los autores, una reducción de la brecha digital que existe entre lectores. Hoy ya es frecuente encontrar estos mapas en la prensa digital para ilustrar y complementar determinadas noticias. Amplían la información porque aportan datos específicos sobre ciertas regiones de una manera estructurada y 


\section{Las nuevas narrativas, en el entorno social Universidad de La Laguna, diciembre de 2019}

ordenada, y esta se muestra en cuanto el usuario coloca el cursor en una determinada zona del mapa.

A veces el mapa no incluye solo este tipo de interactividad. Aunque esta es característica del formato, su intensidad es reducida si la comparamos con otras tipologías del mapa interactivo. Este suele ser más complejo y apostar por una interacción alta por parte del usuario. Esto ocurre cuando se le habilita para colaborar en la propia construcción cartográfica del proyecto. El mapa continúa siendo interactivo, pero suma además una colaboración que se nutre de la lógica de la cultura participativa en la red. Esta origina una multitud de métodos que apuestan por la inclusión del usuario en procesos mediáticos y de producción cultural. Se inicia así un proceso de creación colectiva que incorpora relevantes matices sociales al mapa interactivo, más allá de la información que este presente.

Este potencial del mapa interactivo ha originado una serie de iniciativas de carácter social en diferentes ámbitos, como ya se indicó previamente, lo que da cuenta del impacto que ha tenido el formato.

Destacamos la iniciativa OpenStreetMap, una plataforma de uso libre y licencia abierta para la construcción de mapas de manera colaborativa, así como el proyecto humanitario Missing Maps, desde el que se crean mapas con información sobre regiones del planeta en situación de vulnerabilidad y siempre en colaboración con las poblaciones locales. Por otro lado, la posibilidad de anotación espacial en los mapas digitales pronto dio lugar a plataformas, como Fix My Street y Repara ciudad, que se nutrían de la participación ciudadana de los usuarios que las empleaban para reclamar mejoras locales (Jiménez y Simelio, 2011).

Asimismo, nuevos términos fueron introducidos para definir este fenómeno desde distintas disciplinas, tales como naogeografía, término que hace referencia a la creación de mapas gracias a la participación de la ciudadanía, o "collaborative mapping", que es descrito como "an initiative to collectively produce models of real-world locations online that people can then access and use to virtually annotate locations in space" (Mac Gillavry, 2006: 103). 


\section{Las nuevas narrativas, en el entorno social Universidad de La Laguna, diciembre de 2019}

Desde el periodismo también se ha valorado la idoneidad del mapa colaborativo como herramienta informativa. Cabeceras mediáticas como The Guardian o The New York Times impulsan iniciativas que combinan el periodismo de datos con la interfaz cartográfica. En el marco de los medios convencionales, esto da lugar a una apertura para el periodismo ciudadano.

The Guardian fue responsable, por ejemplo, del proyecto The Counted (2015), una base de datos sobre las personas asesinadas por la policía de los Estados Unidos durante los años 2015 y 2016. El mapa muestra datos como el origen de las víctimas, la fecha, enlaces a las noticias en prensa que informaban del caso o su pertenencia a minorías, lo que permite apuntar a una situación de discriminación. Bajo el reclamo de "help us document every police killing in America", el sitio web alienta la participación de usuarios para generar una estructura colaborativa que ayude a la investigación.

También el ámbito del audiovisual se ha hibridado con frecuencia con este modelo de interfaz. Así, el documental interactivo ha empleado los mapas para construir un retrato diverso de las comunidades que representa. En este sentido, Gifreu (2013: 444) ya incorporaba el mapa dentro de la modalidad de navegación espacial en su segunda propuesta de clasificación de los webdocs en función de la experiencia del usuario: "metáfora gráfica de la recreación digital de un escenario físico descrito o que mantenga relación con el contexto o tema del documental".

Aparte de estas aplicaciones, el mapa interactivo ha encontrado también un fértil desarrollo como instrumento para la investigación en el marco de las humanidades digitales. La cartografía digital ha sido valorada como una herramienta relevante para la construcción de conocimiento mediante la participación ciudadana (Moreno Sardà, Molina Rodríguez-Navas y Simelio Solà, 2017).

A modo de ejemplo, Maltby, Walker y Walsh (2014) describen la base de datos AusCinemas, que forma parte del proyecto de investigación Mapping the Movies. El propósito de AusCinemas era aportar herramientas para evaluar el cambio en el número, naturaleza y distribución geográfica de las salas de cine en Australia durante los veinte años entre 1950 y 1970. Esto permitiría 


\section{Las nuevas narrativas, en el entorno social Universidad de La Laguna, diciembre de 2019}

examinar nuevas hipótesis que pondrían en cuestión algunos de los argumentos que se habían esgrimido tradicionalmente, como la aparición de la televisión. La visualización cartográfica permitía advertir patrones antes desapercibidos, tales como la relación entre la ubicación de las salas o las rutas de transporte público, por ejemplo. En AusCinemas destaca sobre todo la apertura del proyecto. Además de consultarlo, los usuarios pueden aportar imágenes, documentos e incluso historias personales relacionadas con el objeto de estudio, dando lugar a una investigación colaborativa. Mediante una información más exhaustiva y la inclusión de historias alternativas, se facilita una definición del rol social que la institución fílmica tenía en las distintas comunidades.

Un método basado en la recepción del caudal de contenido de los usuarios es el crowdsourcing, una práctica que ya definida en multitud de ocasiones que consiste en el lanzamiento de una convocatoria, por parte de la persona o grupo de personas que impulsen la iniciativa, para la realización de alguna tarea por parte de un grupo de individuos, que puede ser la multitud conectada en un sentido amplio y heterogéneo, pero que también podría ser un colectivo determinado y especializado en función de la tarea que se haya propuesto. Estellés y González (2012) elaboraron una definición integradora de este concepto, en la que indicaban que la colaboración implica un beneficio mutuo, tanto para promotores como para participantes.

El provecho para quien impulse la iniciativa parece evidente y aquí nos centraremos, por tanto, en aquello que obtienen los usuarios. Generalmente, la convocatoria de un mapa interactivo implica la aportación de un contenido, ya sea vídeo, texto o de otro tipo, que se ubica posteriormente en la interfaz cartográfica. La recompensa que obtendrán dependerá de la finalidad del proyecto en concreto, de aquello que se pretenda reflejar en el mapa. Se espera que responda a un beneficio inmaterial, a la adquisición de unas coordenadas virtuales en las que reivindicar una identidad a través de la narración de una experiencia o de la difusión de un trabajo creativo. También que se establezcan vínculos con un determinado colectivo que da forma a la propuesta. El beneficio para los que participan sería el aprovechamiento de ese 


\section{Las nuevas narrativas, en el entorno social Universidad de La Laguna, diciembre de 2019}

proyecto para su expresión personal, para dejar constancia de una representación propia.

Pero, además, el anclaje de esta a un espacio geográfico debería implicar matices vinculados a la pertenencia a un espacio físico y cultural en el que esas personas viven, lo que supone un elemento de valor extraordinario para esa representación. La fundación de ese espacio virtual permitiría la vinculación entre ciertos discursos y el lugar en que se producen, dando información sobre los lugares que habitan los usuarios y sobre las personas con las que comparten territorio.

Nos encontramos, por tanto, ante un formato de comunicación que ha adquirido relevancia en los últimos años y que se ha consolidado como objeto multidisciplinar. Este artículo analizará el mapa interactivo desde el enfoque de la comunicación social, valorando su potencial para la representación plural a través de la multitud de medios que ocupan su contenido. Su objetivo es determinar la capacidad que tiene este formato para expresar la diversidad cultural, con competencia suficiente para distinguirse en un ecosistema de prácticas participativas de diversas intensidades, finalidades y efectos.

\section{Hipótesis y metodología}

Con una metodología de investigación de tipo cualitativo, el artículo se centra en una selección de proyectos significativos que se estructuran a través de la interfaz del mapa interactivo y que proponen su construcción de manera colaborativa: One Day on Earth (2012), Queering the Map (2017) y Radio Right Left (2017). La selección incluye un proyecto temprano y dos de creación reciente, lo que será útil para evaluar una continuidad en las características del formato.

Antes de esto, se ha realizado una consulta bibliográfica que nos ha permitido establecer un sucinto estado de la cuestión del mapa colaborativo. A su vez, esto ha conducido a una definición del formato teniendo en cuenta la manera en que se construye a través de la participación.

Después se ha procedido con el estudio de casos para obtener conclusiones de carácter general sobre la categoría estudiada en relación a dos hipótesis: 


\section{Las nuevas narrativas, en el entorno social \\ Universidad de La Laguna, diciembre de 2019}

En primer lugar, que la unión entre territorialidad simbólica y cultura colaborativa produce una forma de creación colectiva con una potencialidad específica para generar pluralidad, lo que la diferenciaría de tipologías participativas similares.

En segundo lugar, y como resultado del primer punto, que la efectividad del proceso participativo - en tanto proceso de representación de una pluralidad de personas con diversas identidades y referentes culturales- no está determinado solo por la técnica de participación o sus dinámicas, por ejemplo el mencionado crowdsourcing, dado que en ese sentido el mapa colaborativo no encontraría diferencia con respecto a otros formatos. Por el contrario, estaría fuertemente influido por la estética que impone la interfaz.

En este sentido, Scolari (2012: 215) ya indicaba precisamente que "every interface constructs its own user". Es evidente que la experiencia del usuario está mediada por la interfaz. Planteamos por tanto la siguiente pregunta de investigación: ¿de qué manera influye el diseño de la interfaz en la efectividad de una creación participativa con vocación social? Para responderla se ha atendido a dos criterios en el análisis de los proyectos estudiados con la finalidad de determinar la información que desde la propia interfaz cartográfica se expresa: el carácter de las aportaciones y su distribución en los mapas digitales.

\section{El mapa colaborativo como territorio virtual}

Antes se señalaba el carácter desterritorializado de la producción en el espacio virtual, en contraste con el diseño del mapa interactivo, que explicita una materialidad y unas coordenadas. Más allá de ese espacio virtual, la noción de territorio ha sido definida como un "espacio apropiado" (Giménez, 2005). En la misma línea, el fenómeno de los mapas digitales ha sido explicado desde una perspectiva geográfica como una conjunción de "tierra" más "sentido" que da lugar a un "territorio": "aceptamos todas las anotaciones sobre mapas digitales como recursos de expresión de sentidos de lugar válidos en principio (...) como manifestaciones de un orden emergente de producción de significado territorial a través de la web" (Cerda, 2015: 68). 


\section{Las nuevas narrativas, en el entorno social Universidad de La Laguna, diciembre de 2019}

El mapa interactivo conduce a una resignificación simbólica del territorio desde el que cada participante proyecta su mensaje. Esta comunicación, que a veces estará anclada en unas coordenadas específicas del mapa elegidas por el usuario, puede estar destinada a plantear una reivindicación relacionada con ese espacio, a expresar algún sentimiento sobre el mismo o a connotar cuestiones de pertenencia o desarraigo que configuren la experiencia vital de la persona participante. Produce un diálogo con el resto de usuarios pero también con el propio espacio virtual, ya apropiado, convertido en territorio.

Por ese motivo, esas breves comunicaciones a modo de teselas pueden constituir una apertura sobre el terreno, una ventana para su conocimiento. En el ámbito de la geografía, Giménez (2005: 14) explica el interés de la disciplina por hacer un análisis global del entorno. Desde esta perspectiva, se elabora un concepto de paisaje como "traducción visible de un ecosistema". Si el paisaje se define como una perspectiva del territorio desde un determinado lugar, las contribuciones de los usuarios en el espacio virtual del mapa colaborativo, es decir, sus interpretaciones sobre las experiencias en el lugar físico en que se encuentran, podrían constituir una suerte de paisaje virtual. Aunque parcial, una traducción de ese terreno nos permite hacer deducciones sobre el mismo y sobre su traductor.

El espacio del mapa interactivo entendido como un territorio virtual sería entonces un espacio inmaterial pleno de relevancia social, ya que en él tienen lugar interacciones que comunican la identidad y la cultura de miles de usuarios. La materialidad fingida de la representación cartográfica condiciona la producción de contenido porque, anclado a un contexto geográfico determinado, termina por expresar las implicaciones de habitar ese lugar.

\section{Casos de estudio: One day on Earth (2012), Queering the Map (2017) y} Radio Right Left (2017)

Un tiempo antes de que Kevin Macdonald estrenara Life in a Day (2011), el documental One Day on Earth ${ }^{1}$ (dirigido por Ruddick y Litman) ya había sido

1 Consultado en: http://archive.onedayonearth.org/index.php/videos 


\section{Las nuevas narrativas, en el entorno social Universidad de La Laguna, diciembre de 2019}

iniciado. El proyecto consistió en una convocatoria global dirigida a la comunidad conectada para después convertir su contenido en un archivo en internet y en un largometraje. Se pretendía documentar cada región del planeta en tres periodos, correspondientes a los años 2010, 2011 y 2012.

Dada la delimitación de la convocatoria, actualmente no se permiten más contribuciones, pero queda el archivo: formado por alrededor de tres mil horas de vídeo que recibió el proyecto por parte de más de siete mil personas. Contó además con el apoyo del Programa de Naciones Unidas para el Desarrollo, de Human Rights Watch, World Wildlife Fund y Oxfam International. El rango de participantes iba desde niños con que captaban imágenes con cámaras de móviles hasta profesionales (Barnes, 2011).

El mapa se presenta cubierto por círculos verdes que indican el número de contribuciones por zona. Si el usuario clica en cualquiera de estos círculos, se acercará a la región y podrá seleccionar una aportación en concreto. Tras hacerlo, se abrirá una ventana que, sin llegar a cubrir todo el mapa, mostrará el vídeo, el nombre del mismo, el año en que se subió, su autor, una descripción libre que en ocasiones versa sobre el contenido del vídeo, así como los créditos, formato del vídeo e idioma. Se puede compartir, dejar un comentario o ver vídeos relacionados.

En One Day on Earth es muy significativa la variedad estética y temática de las aportaciones, dada la libertad de la convocatoria: desde reivindicaciones sociales, comentarios cotidianos o grabaciones sin voz; y tanto vídeos con montaje sofisticado y formato de entrevista, como grabaciones de una sola toma que retratan algún momento del día. Es, por tanto, un verdadero archivo en el que se mezclan contenidos de una enorme heterogeneidad. Aparte de la geolocalización, que permite la correspondencia del contenido con sus coordenadas, la temporalidad de tres años que caracteriza a este archivo sirve para contextualizar su contenido, lo que a su vez permite sacar más conclusiones sobre el carácter de las aportaciones. 


\section{Las nuevas narrativas, en el entorno social Universidad de La Laguna, diciembre de 2019}

Nuestro segundo caso de estudio es Queering the Map² (2017). Impulsado por el diseñador canadiense Lucas LaRochelle con el objetivo de crear un archivo donde se compartieran experiencias del colectivo LGBT+, aquí la aportación solo puede ser textual y la persona participante podrá elegir las coordenadas en las que la ubica aunque no se encuentre allí. Así, el autor de Queering the Map explica que la web no accede a la ubicación de las personas participantes (Burke, 2018). Tampoco aparecen perfiles, nombres o fotografías de los participantes, lo que refuerza el anonimato e impide las presunciones que pudieran darse sobre la identidad de los participantes. El mapa solo muestra los indicadores donde se ha colocado algún contenido, y tras clicar en ellos aparece el sobrio cuadro de texto.

En el mes de mayo de 2018, Queering the Map ya había recibido más de diez mil contribuciones (Garrido, 2018). El proyecto de LaRochelle se complementa además con una cuenta en Instagram donde se suben capturas de algunos de los testimonios, por lo que funciona como canal de comunicación alternativo que dirige la mirada del usuario hacia contribuciones que su autor quiere destacar.

Con cierta similitud en su formato, nuestro tercer caso de estudio, titulado Radio Right Left' (2017), formula la siguiente pregunta: "how do you feel about the future of America and your place in it?". Su propósito es la apertura de un espacio que permita a los participantes, en este caso mayoritariamente estadounidenses, establecer una comunicación libre y anónima sobre el futuro de los Estados Unidos. Dado que el responsable es un artista sonoro, Halsey Burgund, las contribuciones son archivos de audio.

Además de la limitación en cuanto a la convocatoria - puede participar cualquier usuario, pero el planteamiento del proyecto parece acotarlo a residentes en Estados Unidos_- Radio Right Left también dirige la respuesta de las personas participantes con un listado de categorías entre las que debe elegir aquella con la que sienta mayor identificación personal: hopeful, determined, divided, worried, inspired, other. Tras seleccionar una de ellas, el

\footnotetext{
2 Consultado en: https://www.queeringthemap.com/

3 Consultado en: https://radiorightleft.org/
} 


\section{Las nuevas narrativas, en el entorno social Universidad de La Laguna, diciembre de 2019}

participante deberá también situar su testimonio en la ubicación que mejor le represente, según las instrucciones de la web. Para escuchar los testimonios, se podrán filtrar por categorías, así como prescindir del mapa y atender a un orden aleatorio que supone la pérdida de la ubicación a la que pertenecen los audios.

\section{Los paisajes del mapa colaborativo: resultados y discusión}

Además de permitirnos comprobar la variedad del mapa interactivo como formato, los casos de estudio mostrarán la pertinencia de entender su contenido como paisajes o traducciones visibles del territorio, es decir, de tener adscritos elementos culturales determinados por su ubicación.

Todos los casos tienen una serie de características en común, como son la interfaz cartográfica, la colaboración, el crowdsourcing y la ubicación del contenido en el mapa. Pero cada uno presenta un carácter diferente, no solo en el medio empleado, ya sea texto, sonido o vídeo, sino sobre todo en su finalidad.

Recuperamos los criterios mencionados anteriormente, distribución de los contenidos y carácter de los mismos, para el análisis de los casos estudiados:

1. Distribución de las contribuciones en los mapas digitales. Comprobamos que existe un evidente contraste entre regiones en Queering the Map y en Radio Right Left, mientras que en One Day on Earth la distribución es bastante homogénea a lo largo de todo el mapa. Esto se produce en este último caso por el apoyo institucional con el que contó la iniciativa, como se ha mencionado antes. La promoción y financiación del proyecto habrían permitido una participación elevada y diversa, lo que supone la consecución de uno de los principales objetivos de cualquier iniciativa que emplee una dinámica de crowdsourcing.

En Queering the Map, las contribuciones son muy abundantes en el espacio que corresponde a las regiones de Europa, Canadá, Estados Unidos, Nueva Zelanda, así como en algunos países latinoamericanos, asiáticos y una parte de Australia. En cambio, otras áreas están prácticamente vacías, lo que puede responder, en algunos casos, a una situación de limitación de 


\section{Las nuevas narrativas, en el entorno social Universidad de La Laguna, diciembre de 2019}

derechos del colectivo LGBT+ en determinados países. No hay que dejar de considerar, no obstante, otros posibles factores a tener en cuenta, como un desconocimiento del proyecto o un menor acceso a la red.

El último proyecto es particular, ya que permite alojar contenido desde otras regiones pero está dirigido al mapa de Estados Unidos. Esto complica una distribución variada en el mapa, determinada además por el diseño de la iniciativa. Enunciados encontrados en el sitio web, tales como "we are in this together no matter what, so the more we understand each other, the more we can work together to create a positive future for everyone", parecen reivindicar una identidad nacional que, como tal, no incluye a participantes de otras nacionalidades. Por tanto, no se pueden sacar conclusiones de carácter político o cultural en función de la distribución

2. Carácter de las aportaciones. Queering the Map es el caso en que la participación tiene un sentido más político, social e identitario, lo que se refleja en su contenido. Encontramos textos que aprovechan la interfaz cartográfica para denunciar experiencias de discriminación vinculadas a un lugar ("I lived most of my life here. As I look back on it I always felt gay, but I could only put a label on it when I came to the US. I felt really isolated in Russia as a lesbian") y también para fijar momentos de descubrimiento personal ("me di cuenta que era lesbiana aquí, en esta bellísima ciudad surreal") o de reivindicación sobre la identidad asociada a un territorio donde sus derechos son vulnerados y donde se produce discriminación institucional ("there are queer people here").

El carácter más cercano al activismo de este proyecto implica que, aunque su convocatoria es tan amplia, incorpore un control sobre el contenido que su autor justifica por la eliminación de los mensajes de odio. Así, explica en una entrevista que en cierto momento comenzaron a proliferar mensajes homófobos (Burke, 2018).

También Radio Right Left presenta una vocación reivindicativa a través de la búsqueda de un diálogo que trata de reforzar un sentimiento de comunidad que ha sido debilitado. Pueden escucharse testimonios de preocupación sobre el futuro que vivirán las siguientes generaciones, los 


\section{Las nuevas narrativas, en el entorno social Universidad de La Laguna, diciembre de 2019}

lazos rotos entre conciudadanos, así como críticas a Donald Trump. Aunque las categorías mencionadas antes, que etiquetan todos los contenidos, pueden resultan limitadores, permiten por otro lado conformar una imagen general de las sensaciones predominantes con el simple gesto de navegar por el mapa.

Finalmente, la generalidad de One Day on Earth y su finalidad algo más imprecisa conducen a que el contenido no esté tan definido. Sin embargo, se produce una genuina traducción de los vídeos como paisajes de cada uno de los territorios, gracias al formato audiovisual y a la vocación del proyecto, que anunciaba su intención de "documentar el mundo". Por ello es significativo su valor como archivo de la diversidad cultural a lo largo del planeta. Se encuentran escenas cotidianas, vídeos familiares y también otros con perspectiva de documental de crítica social. En todo caso, los participantes se ven impelidos a hablar sobre el lugar: la interfaz promueve un comentario sobre el territorio.

\section{Conclusiones}

El mapa colaborativo evidencia que la evaluación de la creación participativa no debe ser ajena a un conocimiento preciso de las singulares características de las interfaces, los formatos y los diseños digitales. Desde esta perspectiva, la interfaz del mapa condiciona la participación y promueve la apertura de una conversación múltiple, fragmentada y colectiva acerca de los territorios que configuran las experiencias vitales de los participantes. Esto diferencia este formato participativo de tantas otras modalidades que tienen lugar en la red. No es solo el proceso o la herramienta de participación el que da forma a la comunicación, sino la propia interfaz que, más allá de sus valores estéticos, también incorpora matices semánticos.

Al condicionar el sentido del mensaje, el mapa colaborativo genera también una participación distintiva, ya que la comunicación queda marcada por la vivencia de la persona que participa, lo que ocasiona que refleje una diversidad de perspectivas significativa, basadas en experiencias o enfoques personales. 


\section{Las nuevas narrativas, en el entorno social Universidad de La Laguna, diciembre de 2019}

De esta manera, la participación proyecta, como se ha visto en los casos de estudio, un lugar geográfico pero, sobre todo, el significado de habitar ese lugar: condiciones materiales, contexto político, perspectivas de avance o involución social, así como sentimientos de arraigo o desapego que el territorio pueda generar.

Por otro lado, la atención a la distribución de contenido a lo largo del mapa nos permite percibir que, cuanta más vocación política e identitaria tiene la iniciativa, más radical será el contraste de la participación entre regiones. Esto implica que el formato en sí mismo ofrecerá información implícita sobre los territorios, y no solo a través de la comunicación directa de los usuarios.

En definitiva, la conversación desde un territorio en el espacio virtual determina la participación, ya que con esta la comunidad transmite mensajes que actúan como traducción del lugar geográfico en que viven o en el que han tenido experiencias relevantes, con las implicaciones que esto puede tener en la construcción de la identidad de los individuos. Esta modalidad de creación participativa constituye, en ese sentido, una singular forma de expresión de la diversidad que habilita además a la comunidad conectada para aprovechar una característica vía de reivindicación social y cultural ante la multitud que la escucha.

\section{Referencias bibliográficas}

J Alberich-Pascual y F Gómez-Pérez (2017): "Tiento para una estética transmedia. Vectores estéticos en la creación, producción, uso y consumo de narrativas transmediales", en Tropelías. Revista de Teoría de la Literatura y Literatura Comparada, 28, páginas 9 a 20.

B Barnes (2011): "Filmmakers' First Opus Stars Everyone on Earth", en The New York Times, agosto: https://nyti.ms/2qRNIB2 (12-11-2019).

S Burke (2018): "Find Yourself in the Queer Version of Google Maps", en Vice, abril: https://bit.ly/2pj706F (12-11-2019) 


\section{Las nuevas narrativas, en el entorno social \\ Universidad de La Laguna, diciembre de 2019}

D Cerda (2015). "Mapas digitales y sociedad: geosemántica social, el poder del sentido de lugar", en Polígonos. Revista de Geografía, 27, páginas 61 a 96.

E Estellés-Arolas y F González-Ladrón-de-Guevara (2012): "Towards an integrated crowdsourcing definition", en Journal of Information Sciencie, 38 (2), páginas 189 a 200.

M Garrido (2018): "El mapa queer que desafía las reglas sociales de sexo y género", en Yorokobu, mayo: https://www.yorokobu.es/queering-the-map/ (1211-2019).

A Gifreu (2013): El documental interactivo como nuevo género audiovisual. Estudio de la aparición del nuevo género, aproximación a su definición y propuesta de taxonomía y de modelo de análisis a efectos de evaluación, diseño y producción (tesis doctoral). Universidad Pompeu Fabra, Barcelona.

G Giménez (2005): "Territorio e identidad. Breve introducción a la geografía cultural", en Trayectorias, 7 (17), páginas 8 a 24.

D Jiménez y N Simelio (2011): "Mapas digitales y participación ciudadana”, en Actas III Congreso Internacional Latina de Comunicación Social. La Laguna (Tenerife): Universidad de la Laguna.

E Mac Gillavry (2006): "Mapping and GIS: An Alternative Geographic Information Framework". En VV. AA., Collaborative Geographic Information Systems (Coords., S Balram y S Fraser). Hershey, London: Idea Group Publishing.

R Maltby, D Walker y M Walsh (2014): "Digital Methods in New Cinema History". En VV. AA., Advancing Digital Humanities. Research, Methods, 


\section{Las nuevas narrativas, en el entorno social Universidad de La Laguna, diciembre de 2019}

Theory. (Eds., P L Arthur y K Bode). Houndmills, Basingstoke: Palgrave Macmillan.

A Moreno Sardà, P Molina Rodríguez-Navas y N Simelio Solà (2017): "CiudadaniaPlural.com: de las Humanidades Digitales al Humanismo Plural", en Revista Latina de Comunicación Social, 72, páginas 87 a 113.

C A Scolari (2012): "Media Ecology: Exploring the Metaphor to Expand the Theory", en Communication Theory, 22, páginas 204 a 225.

L Renó y D Renó (2013): "Narrativa transmedia y mapas interactivos: periodismo contemporáneo", en Razón y Palabra, 83.

D Renó y L Renó (2015): "Mapas interactivos como plataformas informativas para ciudadanos desconectados", en Razón y Palabra, 89. 